\title{
Surface micromachined mechanisms and micromotors
}

\author{
Mehran Mehregany $\dagger$ and Yu-Chong Taił \\ tElectronics Design Center, Department of Electrical Engineering and Applied \\ Physics, Case Western Reserve University, Cleveland, OH 44106, USA \\ $\ddagger$ Department of Electrical Engineering, California Institute of Technology, \\ Pasadena, CA 91125, USA
}

Received 20 February 1991, accepted for publication 1 April 1991

\begin{abstract}
Electric micromotors are sub-millimeter sized actuators capable of unrestrained motion in at least one degree of freedom. Polysilicon surface micromachining using heavily phosphorus-doped LPCVO polysilicon for the structural material, LPCVD silicon nitride for the electrical isolation and deposited silicoñ dioxide for the sacrificial material has formed the fabrication technoiogy base for the development of these micromotors. Two polysilicon surface micromachining processes, referred to here as the center-pin and flange, have been demonstrated for the fabrication of passive mechanisms and micromotors. Passive mechanisms such as gear trains, cranks and manipulators have been implemented on silicon. Reported operational micromotors have been of the rotary variable-capacitance salient-pole and harmonic (or wobble) side-drive designs. These micromotors are capable of motive torques in the $10 \mathrm{pN} \mathrm{m}$ order of magnitude range. Preliminary progress has been made in studying the operational, friction and wear characteristics of these micromechanical devices. Typical operational voltages have been as low as $37 \mathrm{~V}$ and $26 \mathrm{~V}$ across $1.5 \mu \mathrm{m}$ air gap salient-pole and harmonic micromotors. These excitations correspond to electric

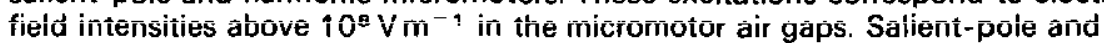
wobble micromotors have been reported to operate at speeds as high as $15000 \mathrm{rpm}$ and $700 \mathrm{rpm}$, respectively. Micromotor lifetimes of at least many milions of cycles over a period of several days have been reported. Friction and wear are important in micromotors; studies aimed in understanding them are discussed in the paper. New designs, materials, fabrication techniques, and applications are expected to play a key role in the future development and progress of the micromotor technology described here.
\end{abstract}

\section{Introduction}

A necessary precursor to the development of monolithic micromechanical systems is the fabrication of micromechanical components and devices. Microfabrication provides a powerful tool for batch processing and miniaturization of mechanical systems into a dimensional domain not accessible by conventional machining. Furthermore, microfabrication provides the potential for integration of mechanical systems with the associated electronics required for closed-loop control. Integrated fabrication techniques eliminate the need for discrete component assembly as has been required in other studies [1-4]; hence, further dimensional control, including component size and intercomponent clearance, is limited only by the processing technology.

Integrated fabrication techniques for passive micromechanisms using polysilicon surface micromachining were first demonstrated by the authors and their col- leagues at AT \& T-Bell Laboratories $[5,6]$ and at the University of California-Berkeley $[7,8]$. Similar research at MIT [9-11] identified many potential problems encountered in the development of an electric microactuator (micromotor) technology. The initial work at Berkeley led to the development of the first operational electric micromotor [12,13].

This paper presents a review of the electric micromotor technology from the start to the present. We begin with the developed microfabrication technology base by describing the basic polysilicon surface micromachining processes for the fabrication of passive polysilicon mechanisms. Next, the electric micromotor technology is discussed and reported micromotor designs are described. Critical issues related to the fabrication of micromotors as well as micromotor operational, friction and wear characteristics are discussed. Finally, a brief discussion of current limitations and future trends is presented. 


\section{Passive mechanisms}

Traditionally, bulk and surface micromachining of silicon have been used to fabricate a variety of micromechanical structures such as thin silicon diaphragms $[14,15]$, beams $[16-18]$ and other suspended structures [19-22] in single-crystal silicon or in films deposited on a silicon substrate. These micromechanical structures are generally limited in motion to small deformations and are physically attached to the substrate. Such elastic components may be used occasionally as flexible joints, but their overall usefulness in the design of mechanisms is limited. A 'mechanism' as used here is a means for transmitting, controlling or constraining relative movement and is considered as a collection of rigid bodies connected together by joints.

This section describes the extension of conventional surface micromachining techniques to the integrated fabrication of passive planar polysilicon mechanisms incorporating lower and higher kinematic pairs. The surface micromachining technology described here provides the basis for the fabrication of the electric micromotors below. Two variations of a two-level polysilicon process, referred to as the center-pin and the flange processes, are described that are appropriate for the implementation of the two lower kinematic pairs (i.e., revolute and prismatic) commonly used in macroscopic mechanical systems. In this paper, we have used the term bearing when a lower kinematic pair is used to support continuous motion.

\subsection{Surface micromachining}

Surface micromachining relies on encasing specific device structural parts in layers of a sacrificial material during the fabrication process. The sacrificial material is then dissolved away in a chemical etchant that does not attack the structural parts. This technique was first demonstrated by Nathanson et al [23] in building a free standing metal-gate field-effect transistor.

Surface micromachining requires a compatible set of structural materials, sacrificial materials and chemical etchants $[17,24,25]$. The structural materials must possess the desired electrical and mechanical properties for the application in mind. The sacrificial materials must possess the proper mechanical properties so as not to cause device failure during the fabrication process. The chemical etchants must be able to preferentially etch the sacrificial materials with respect to the structural parts, must have proper viscosity and surface tension characteristics to enable them to fully remove the sacrificial layers, and must not leave residues behind.

For monolithic micromechanical systems, it is desirable to have structural materials with good mechanical properties including no residual stress, very high modulus, high fracture strain, very low frictional coefficient and high wear resistance. Due to the actuator requirements, good conductors and good insulators are required for the structural materials; however, it is more desirable to be able to tailor the material conductivity in the range of these two extremes. Finally, the material candidates should be compatible with integrated circuit (IC) technology.

Polysilicon surface micromachining using doped or undoped polysilicon as the structural material and silicon dioxide as the sacrificial material forms the backbone of the technology described in this paper. Polysilicon surface micromachining is the best documented $[16-18,26]$ surface micromachining technique which most closely meets many of the requirements described above. Two variations of a two-level polysilicon process, referred to as the center-pin and the flange processes, are described below and have been used for successful fabrication of mechanisms and micromotors on silicon. These basic processes (or minor modifications of them) can also be used in conjunction with structural materials other than polysilicon.

\subsection{The center-pin process}

The center-pin process is described here in its general form. To demonstrate the general center-pin design, consider the implementation of a rotor which is free to turn about a center bearing. The basic process uses two polysilicon and two silicon dioxide depositions as well as four photolithography steps $[6,8]$.

Figure 1 shows cross-sectional views of a rotor on a center-pin bearing during the fabrication process. After a blanket deposition of silicon dioxide, the bushing molds are time etched to a desired depth in the oxide (figure 1(a)). After a blanket deposition of polysilicon, the rotor

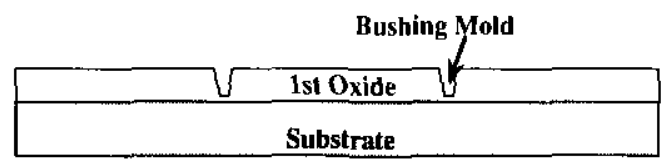

(a)

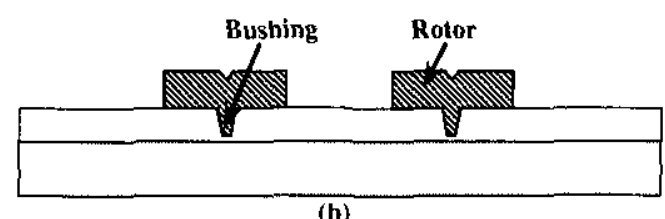

(b)

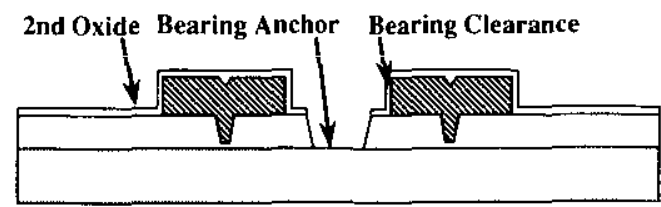

(c)

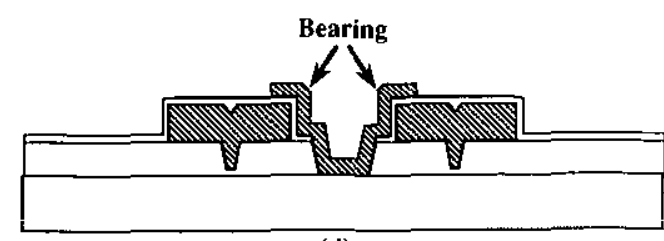

(d)

Figure 1. The general center-pin fabrication process: (a) After the bushing mold is patterned; (b) after the rotor is patterned; (c) after the bearing anchor is patterned; and (d) completed device. 
is patterned (figure $1(b)$ ) by reactive-ion etching (RIE). Note that the bushings are formed automatically. The bushings are an integral part of the center-pin bearing design and eliminate rotor to substrate stiction problems in the final device [6]. After a second blanket deposition of silicon dioxide, the bearing anchor is opened (figure 1(c)). After a second blanket deposition of polysilicon, the center-pin bearing is defined (figure 1(d)). At this point, the sacrificial silicon dioxide is dissolved in hydrofluoric acid (HF) to release the rotor. When released, the bushing rests on the silicon substrate, reducing the surface area of contact between the rotor and the substrate. During operation, the bushing slides on the substrate.

Figure 2 is a SEM photograph of a gear-train with 1.4:1.0:1.0 gear ratios, fabricated in the center-pin process, after it has been released. The smaller gears are $125 \mu \mathrm{m}$ in diameter. The first and second polysilicon layers are $4.5 \mu \mathrm{m}$ and $3 \mu \mathrm{m}$ thick, respectively. The first and second sacrificial oxide are $4 \mu \mathrm{m}$ and $1 \mu \mathrm{m}$ thick, respectively. The bushing mold is etched to a depth of $2 \mu \mathrm{m}$.

The center-pin process has the capability of producing a self-aligned or a non-self-aligned bearing. Let $s_{1}$ and $s_{2}$ denote the thicknesses of the first and second oxide sacrificial layers, respectively. Let $p_{1}$ and $b$ denote the thickness of the first polysilicon layer and the height of the bushing. Then if $p_{1}+b<s_{1}+s_{2}$, the rotor can slide under the bearing, resulting in a non-self-aligned bearing. The bearing clearance for the non-self-aligned case, is the difference between the radius of the top of the bearing anchor opening and the rotor inner radius. This clearance can be large and is governed by the mask dimensions as well as the slope of the bearing anchor sidewalls produced by the fabrication process. If $p_{1}+b>s_{1}+s_{2}$, the bearing clearance is specified by the oxide thickness on the sidewalls of the rotor inner radius. This results in a self-aligned bearing, which is the type used for all of the center-pin bearing micromotors to date.

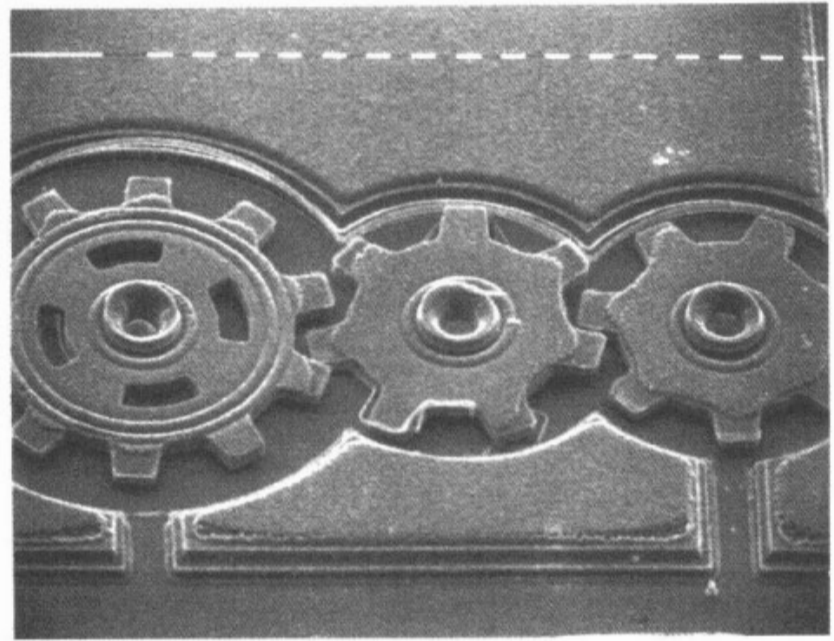

Figure 2. A released gear train fabricated in the centerpin process [6],

\subsection{The flange process}

The flange process is described here in its general form. To demonstrate the general flange design, consider the implementation of a rotor which is free to turn about a center bearing. The basic process uses two polysilicon and two silicon dioxide depositions as well as four photolithography steps [8]. The overall process is very similar to the center-pin process and uses only minor modifications to accomplish the flange bearing. For the same number of film depositions, the flange process provides more flexibility in design as compared with the center-pin process. However, the center-pin process is somewhat simpler.

Figure 3 shows cross-sectional views of the rotor on a flange bearing during the fabrication process. After a blanket deposition of silicon dioxide, a blanket layer of polysilicon is deposited. The rotor is patterned into that polysilicon layer (figure 3(a)) by RIE. At this point, the oxide at the inner radius of the rotor is underetched (figure 3(b)). After a second blanket deposition of silicon dioxide, the bearing anchor is opened (figure 3(c)). After a second blanket deposition of polysilicon, the bearing is defined (figure 3(d)). Note that the flange in the bearing forms automatically and functions in a similar manner to the bushings in the center-pin bearing design. At this point, the sacrificial silicon dioxide is dissolved in HF to release the rotor. When released, the rotor rests on the bearing flange and does not contact the substrate, reducing surface area of contact. During operation, the rotor slides on the flange. Note that the flange bearing is
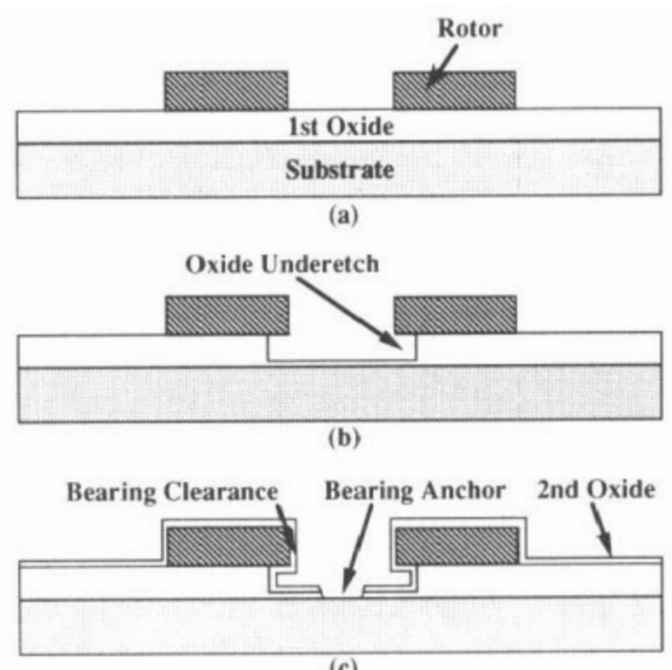

(c)

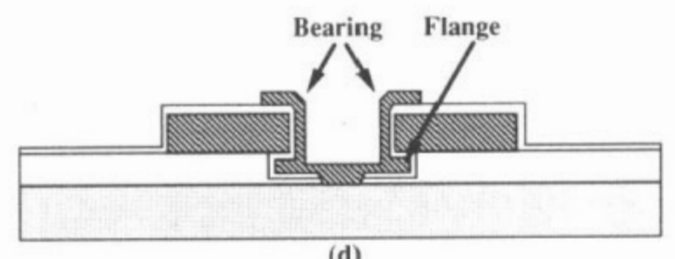

(d)

Figure 3. The general flange fabrication process: (a) After the rotor is patterned; (b) after the oxide underetch; (c) after the bearing anchor is patterned; and (d) completed device. 


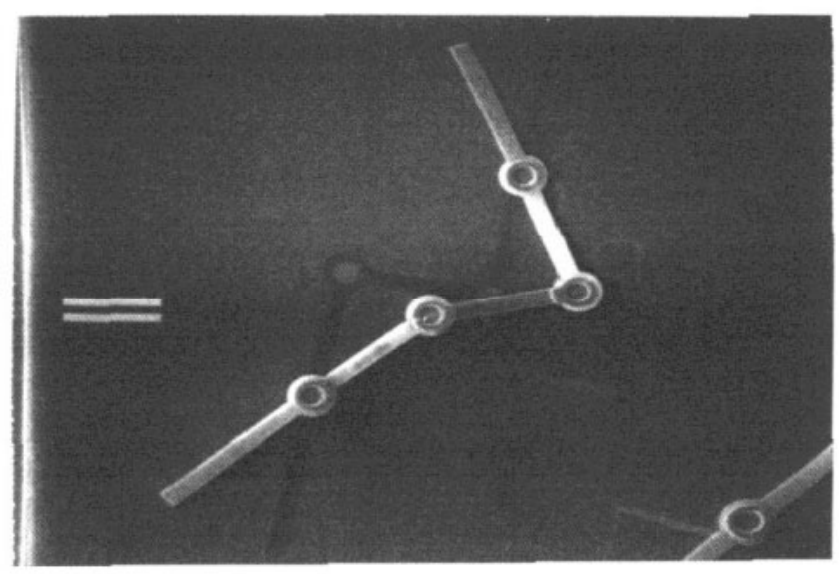

Figure 4. A released four-joint crank mechanism fabricated in the flang process [8].

always self-aligned and the bearing clearance is specified by the thickness of the oxide on the rotor inner sidewalls.

Figure 4 shows an entirely released four-joint crank mechanism fabricated in the flange process. The first and second polysilicon layers are $1.5 \mu \mathrm{m}$ and $1 \mu \mathrm{m}$ thick, respectively. The first and second sacrificial layer oxide are $1.5 \mu \mathrm{m}$ and $0.5 \mu \mathrm{m}$ thick, respectively. The flange extends by $2 \mu \mathrm{m}$ under the first polysilicon layer components. The basic flange process above has also been used to fabricate a three-degrees-of-freedom mechanism on silicon [27].

\section{Electric micromotors}

The previous section described polysilicon surface micromachining techniques suitable for the fabrication of passive mechanisms on silicon. However, microelectromechanical systems require large-motion actuators to power the linkages. Electric, as opposed to magnetic, drive has been argued as being preferable in the microscopic scale $[9,28]$. This is mainly due to two factors. First, the electric breakdown limit in air increases drastically for microscopic (order of $1 \mu \mathrm{m}$ ) air-gap sizes. Typically, the electric field breakdown limit in air is near $3 \times 10^{6} \mathrm{~V} \mathrm{~m}$ for macroscopic dimensions and increases to above $10^{8} \mathrm{Vm}$ for microscopic air-gap sizes [29]. However, for magnetic actuators, the magnetic saturation limit is independent of actuator size. Therefore, for microscopic sizes, the stored energy density limits for electric and magnetic actuators become comparable and are of the order of $10^{5}-10^{6} \mathrm{~J} \mathrm{~m}^{-3}$. Second, required materials for electric actuators, namely conductors and insulators, are available in the IC industry while those for magnetic actuators (i.e., magnetic materials) seldom are. This section concentrates on the electric micromotor technology. The micromotors under discussion are surface micromachined using heavily phosphorus-doped LPCVD polysilicon for the structural material, deposited oxide for the sacrificial layers, and LPCVD silicon nitride for electrical isolation. The micromotor fabrication pro- cesses are based on the center-pin and flange processes of sections 2.2 and 2.3 .

\subsection{Principles of operation}

In general, in an electric actuator, the attractive and repulsive forces generated by electric charge distributions are used to convert electrical to mechanical energy. By proper commutation of these charge distributions on a set of stationary electrodes, known as the stator, and a set of moving electrodes, known as the rotor, continuous motion of the rotor can be achieved. Similar to their magnetic counterparts, electric motors can be categorized based on the specific mechanism of electric actuation. For example, electric induction motors [30] are the counterparts of magnetic induction motors while permanent electret motors are the counterparts of permanent magnet motors. For a more detailed discussion of this topic and an extended bibliography, the reader is referred to $[9,31]$.

To date, only rotary variable-capacitance micromotors (which are the counterpart of variable-reluctance magnetic motors) have been reported because of their design and material requirement simplicity. The operation of these micromotors relies on the storage of electrical energy in a variable rotor-stator capacitance. The change in this capacitance in the direction of motion is proportional to the output torque of the micromotor. In terms of design, one endeavors for optimum torque generation by enhancing the capacitance change in the direction of motion. In terms of material requirements, only good conductors and insulators are required. The material requirements are compatible with polysilicon surface micromachining which has been used almost exclusively for micromotor fabrication so far. However, other fabrication processes are now starting to emerge [32]. Rotary instead of linear designs have been emphasized so far because rotary motors provide force-totorque leverage as compared with linear ones. Furthermore, rotary motion is more suitable for motor diagnostic and characterization studies. For example, rotary micromotors can be spun with an air jet as well as electricity.

\subsection{Reported designs}

Reported micromotors have so far been based on variable-capacitance designs. For the sake of brevity, we will drop the variable-capacitance term in describing the micromotors below. Two micromotor designs based on a top-drive $[9,10,33]$ and a side-drive $[12,13,33,34]$ configuration have been reported. Initially, the top-drive design was considered because of its superior motive torque characteristics. However, top-drive micromotors demonstrated significant design and fabrication shortcomings, namely rotor instability problems and planarization requirements [33]. These shortcomings motivated the development of side-drive micromotors $[12,13,33,34]$. The side-drive design eliminates the rotor instability problems and the need for planarization. 


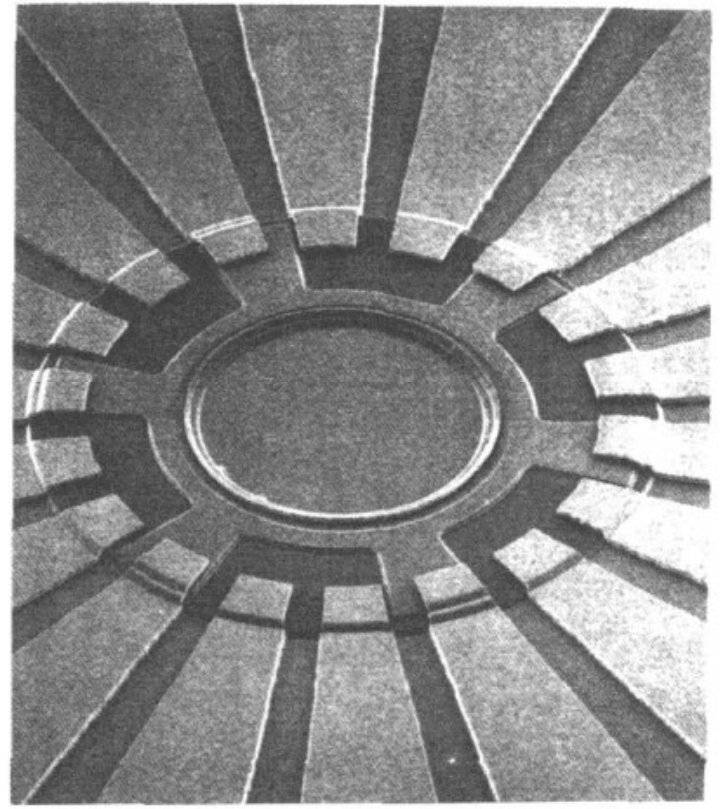

Figure 5. A $300 \mu \mathrm{m}$-diameter, salient-pole, top-drive micromotor [39]. The air gaps are $2 \mu \mathrm{m}$ wide.

However, this is achteved at the expense of sacrificing motive torque.

Figure 5 is a SEM photograph of a typical three-phase, salient-pole, top-drive micromotor. This micromotor is fabricated using a two-level center-pin polysilicon process identical to that in section 2.2 with one added step. The added step is a blanket deposition of a LPCVD silicon nitride isolation layer on the starting silicon substrate. The silicon nitride addition is required for electrical isolation of the stator poles. Note that the stator is fabricated from the second polysilicon deposition and does not require additional process steps. For the micromotor in figure 5 , both polysilicon layers are $2.5 \mu \mathrm{m}$ and are heavily doped (i.e., a sheet resistivity of $7 \Omega$ per square) with phosphorus. The sacrificial layers are deposited from low-temperature oxide (LTO). The first LTO thickness is $2.3 \mu \mathrm{m}$, while the second LTO thickness is $1 \mu \mathrm{m}$; the bushing height is $1.8 \mu \mathrm{m}$.

In the top-drive micromotor design, electric energy is stored in the air gap which is typically $1-2 \mu \mathrm{m}$ and is formed by the overlap of the stator and rotor poles. The air gap in the top-drive micromotor is defined by the two LTO thicknesses and the rotor bushing depth, all of which are easy parameters to control. The micromotor operation relies on tangential electrostatic forces tending to align the rotor poles under the excited stator poles. For micromotor operation, the substrate is electrically grounded while a three-phase bi-polar excitation is applied to the stator. In bi-polar excitation, opposite stator poles of the same phase are excited with equal voltage magnitudes but opposite polarities.

Due to its significantly larger rotor-stator capacitance changes with rotor position, the top-drive micromotor design can provide a large motive torque as compared with that provided by the side-drive designs presented below. However, associated with the rotorstator and the rotor-substrate capacitances, there are large vertical forces tending to clamp the rotor up to the stator poles or down to the substrate. In the former case, the micromotor is destroyed by the large current through the rotor due to the shorting out of the stator. In the latter case, the micromotor will not operate due to the large frictional forces associated with rotor-substrate clamping. Rotor instability is a severe limitation of this design.

Furthermore, since the stator poles are not all located over rotor poles during device fabrication, they are not at the same height (see figure 5). To resolve this problem, an excessively thick second LTO deposition and a subsequent planarization would be required. Otherwise, the stator poles fabricated over rotor poles are at a higher elevation than the remaining stator poles. The planarization requirement is another severe limitation of the top-drive micromotor design. These difficulties have proved the top-drive micromotor design to be impractical. Therefore, research has focused on the design, fabrication and characterization of salient-pole and harmonic (also known as wobble) side-drive micromotors. These sidedrive micromotor designs overcome the design and fabrication difficulties associated with the top-drive micromotors but at the cost of sacrificing output torque.

The salient-pole and harmonic side-drive micromotors are hereafter referred to as salient-pole and wobble micromotors. For the sake of brevity, the sidedrive term is dropped since all operational micromotors to date have been of the side-drive design. We will, however, use the term side-drive micromotors to refer to both the salient-pole and wobble micromotors collectively. Figure 6 is a SEM photograph of a three-phase, salientpole micromotor. Figure 7 is a SEM photograph of a wobble micromotor. The micromotor in figure 6 $[13,35,36]$ is fabricated using the basic flange process of section 2.3 , while that in figure $7[37-39]$ is fabricated using the basic center-pin process of section 2.2 . In both cases, substrate isolation process steps have been added

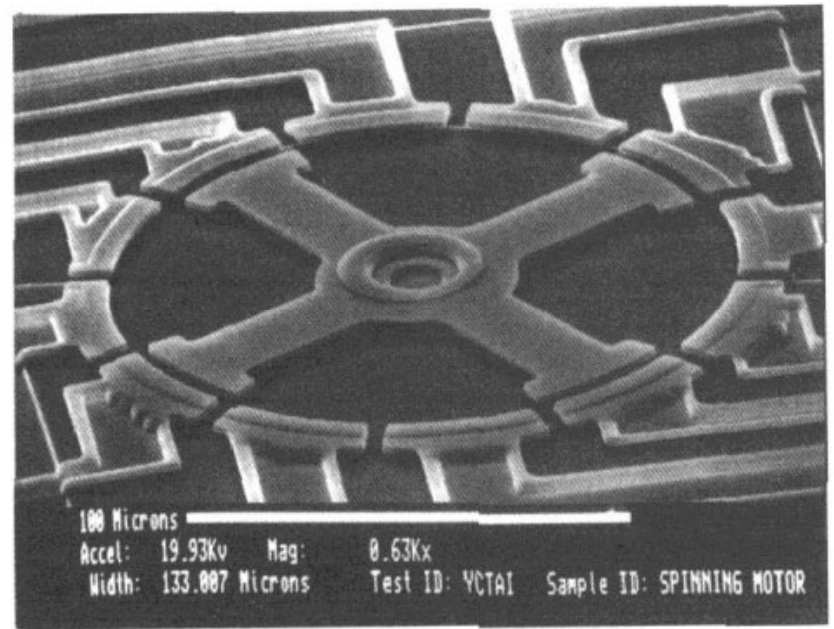

Figure 6. A $120 \mu \mathrm{m}$-diameter, salient-pole, side-drive micromotor [36]; the air gaps are $2 \mu \mathrm{m}$ wide. 


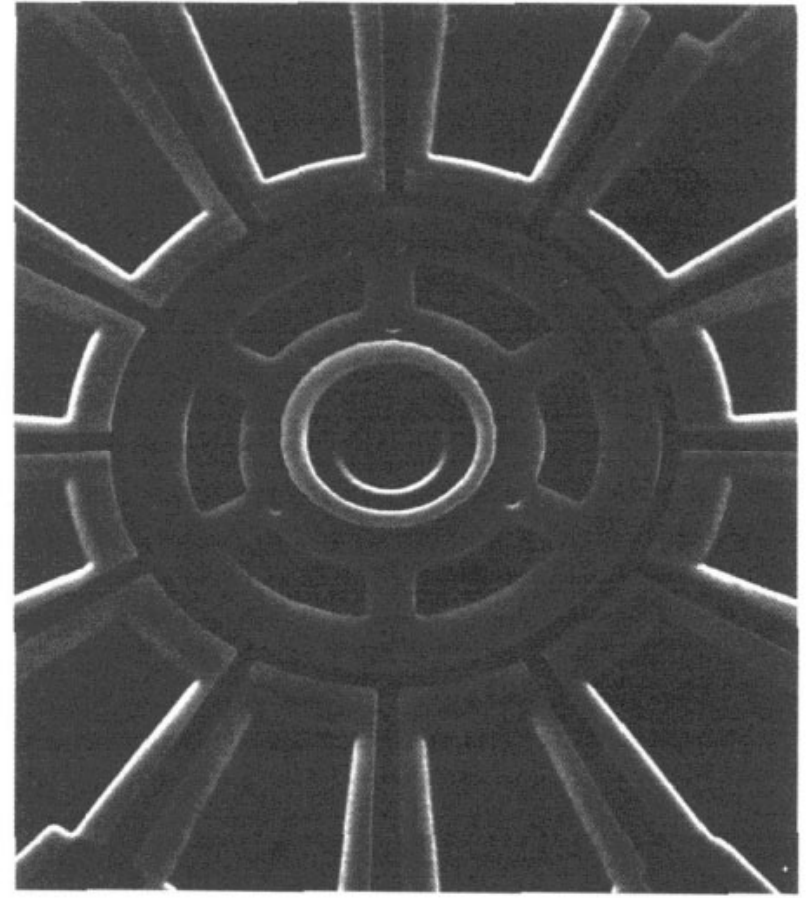

Figure 7. A $100 \mu \mathrm{m}$-diameter, harmonic, side-drive micromotor [39]; the air gaps are $2.5 \mu \mathrm{m}$ wide

to the basic fabrication processes. Note that it is possible to fabricate both micromotor types with either of the two bearing designs $[37,39]$.

For the salient-pole micromotor of figure 6, the rotor and bearing polysilicon are $1.5 \mu \mathrm{m}$ and $1 \mu \mathrm{m}$, respectively. The first and second sacrificial oxide layers are $2.2 \mu \mathrm{m}$ and $0.5 \mu \mathrm{m}$ thick, respectively. A $340 \mathrm{~nm}$-thick silicon nitride layer is used to cover the rotor inner radius sidewalls, thereby providing a solid lubricant. For the wobble micromotor of figure 7 , the rotor and bearing polysilicon are $2.2 \mu \mathrm{m}$ and $1 \mu \mathrm{m}$ thick, respectively. The first and second sacrificial oxide layers are $2.3 \mu \mathrm{m}$ and $0.3 \mu \mathrm{m}$ thick, respectively. The bushing height is $1.8 \mu \mathrm{m}$. For both micromotors, a thick sandwich of silicon nitride over silicon dioxide is used for electric isolation of the substrate.

In the side-drive micromotors, electric energy is stored in the gap formed between the rotor and stator sidewalls. Note that capacitance changes with rotor position are significantly smaller for the side-drive micromotors as compared with that for the top-drive design above. This results in a significantly smaller motive torque for the side-drive micromotors. In contrast to the top-drive design, in which the air-gap dimension is controlled by deposited film thicknesses, the air-gap dimension in the side-drive micromotors is controlled by photolithography and pattern delineation; hence, it is more difficult to control.

A conductive polysilicon shield beneath the rotor and in electrical contact with it has been suggested $[36,39]$ to be effective in electrostatically shielding the rotor from

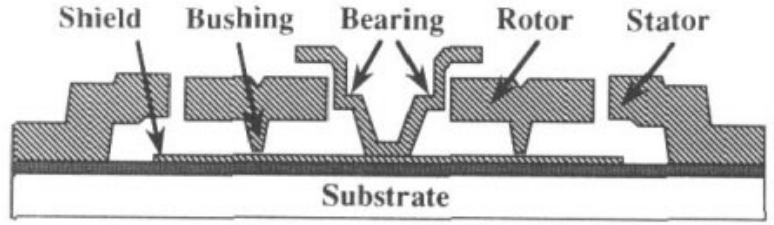

Figure 8. A cross-sectional schematic of the micromotor in figure 7.

the substrate as well as providing a means for biasing the rotor. Figure 8 is a schematic cross-sectional view of the micromotor in figure 7 , demonstrating the polysilicon shield incorporated beneath the rotor. During motor aperation, the hushing slides over the shield. This mechanical contact is intended to provide the required electric contact between the rotor and the shield.

For the salient-pole micromotor operation, the shield is electrically grounded while a three-phase bi-polar excitation is applied to the stator. Micromotor operation relies on tangential electrostatic forces tending to align the rotor poles with the excited stator poles. The salientpole micromotors have been fabricated $[37,39,40]$ with stator to rotor pole number ratios of 3:1 (similar to that in figure (6), 3:2 and $2: 1$. The former two require threephase excitation while the latter requires two-phase excitation. The $3: 2$ architecture provides superior torque coverage in comparison with the other two architectures $[37,39,40]$.

Operating principles of wobble motors have been described in detail $[33,37-39,41,42]$. Figure 9 shows a schematic plan view of a non-microfabricated wobble motor $[4,41,42]$. For this design, motor operation relies on normal electric forces attracting the rotor, which is electrically grounded, to an electrically excited stator pole. Some form of rotor-stator insulation is required since the rotor contacts the excited stator poles. As the electric excitation travels around the stator poles, the rotor follows and rolls on the stator. Since the rotor circumference is smaller than that of the stator, the rolling action produces rotor rotation about its center. The rotor rotational speed is proportional to the electric signal frequency; the proportionality constant is called

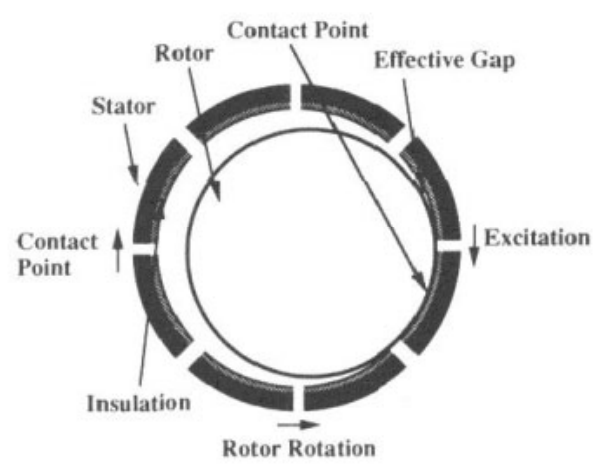

Figure 9. Plan view schematic of a conventional wobble motor. 
the motor gear ratio. A significant advantage of the wobble motor is that its drive torque is proportional to the motor gear ratio. The gear ratio relates the angle of rotation of the stator excitation to the angle of rotation of the rotor; it is equal to the excitation speed divided by the rotor speed.

The central feature of the wobble micromotor is that the rotor wobbles around the center bearing post in contrast to the outer stator in non-microfabricated wobble motors. A schematic plan view of the wobble micromotor design is shown in figure 10. For micromotor operation, the rotor is electrically grounded by externally grounding the shield, and the stator poles are excited sequentially. Micromotor operation relies on normal electric forces attracting the rotor to the excited stator pole. The center bearing limits lateral rotor motion and prevents it from contacting the excited stator pole and shorting out. By ensuring a bearing clearance smaller than the nominal air-gap size, the need for insulation between the rotor and the stator is eliminated. The rotor lateral movement (i.e., wobble distance) is equal to the clearance in the bearing which is the difference between the bearing and inner rotor radii. As the electric excitation travels around the stator poles, the rotor rolls on the bearing. Since the rotor circumference is larger than that of the bearing, the rolling action also produces rotor rotation. The rotor rotational speed is proportional to the electric signal frequency by the gear ratio. In this design, the rotor lateral movement (i.e., wobble distance) is equal to the clearance in the bearing which is the difference between the bearing and inner rotor radii. By ensuring a bearing clearance smaller than the nominal air-gap size, which is typically greater than $1.5 \mu \mathrm{m}$, the need for insulation between the rotor and the stator is eliminated. For the wobble micromotor in figure 7 , the bearing clearance is $0.3 \mu \mathrm{m}$ while the air gap is $2.5 \mu \mathrm{m}$.

For comparable geometrical designs, the wobble micromotors provide higher motive torque than the salient-pole micromotors. This results from the fact that the operation of the wobble micromotors relies on normal electric forces of attraction rather than the tangential component in the salient-pole micromotors. Additionally, the inherent gear ratio in the wobble micromotors is a significant advantage. However, in the

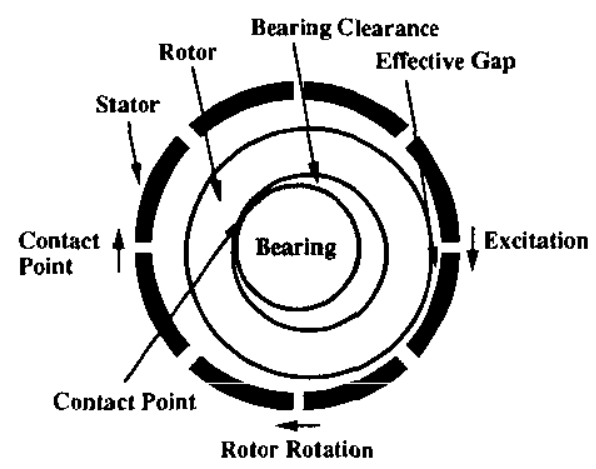

Figure 10. Plan view schematic of the wobble micromotor. wobble micromotors the rotor wobbles as it turns which may be a disadvantage in some applications.

\subsection{Fabrication issues}

Micromotor fabrication has almostit exclusiveiy been based on the center-pin or flange processes of section 2 with the addition of a few steps to provide the shield, stator interconnects and substrate isolation. Fabrication process details for the polysilicon micromotors have been documented previously [12, 13, 33-37, 39, 40, 43-45] and are not repeated here. Instead, we discuss two most critical issues, namely pattern definition and final release, in the micromotor fabrication processes.

The micromotor fabrication requires deposition of films that are thick by $i C$ standards. As these films are deposited and patterned in the course of the fabrication process, the wafer surface topography increases, complicating the pattern definition processes. For large wafer topographies, a thicker resist coating is required to provide proper step coverage and sufficient etch-mask thickness. However, thicker resist coatings compromise the achievable linewidth resolution. For example, for the side-drive micromotors above, surface topography is typically over $2 \mu \mathrm{m}$ at the air gap definition step, requiring a correspondingiy thick resist coating and thereby limiting the minimum air gap size possible. Similar limitations place a lower bound on the bearing radius and bearing clearance dimensions. This in turn results in a degradation of the -salient-pole micromotor performance. Holding all other micromotor parameters constant, larger air gaps lead to reduced motive torque in all of the micromotors above. For the salient-pole micromotors, large bearing radii and bearing clearances lead to increased bearing frictional torque. A bearing radii increase results in a larger lever arm for the bearing frictional force while a bearing clearance increase results in a larger bearing frictional force by increasing the sidepull force (see section 3.5) in the bearing. Finally, more complicated pattern definition processes become necessary for micromotor fabrication as the surface topography increases. For example, at the bearing pattern definition step, the surface topography can be as high as $5 \mu \mathrm{m}$, requiring a tri-level resist process for best results. A LOCOs-based micromotor process has been reported which is a derivative of the center-pin process and reduces topography during micromotor fabrication $[34,40,45]$.

Proper micromotor release is not only required for micromotor operation but it affects the micromotor performance $[37,39]$. However, our current knowledge of the effect of the release process on the micromotor operational characteristics is limited. One critical issue in the micromotor release is the role of native oxide formation on the rotor, bearing, and shield polysilicon surfaces in disrupting proper electrical contact between these respective parts $[37,39]$. If the rotor is not in electrical contact with the shield (see figure 8), the resulting electrostatic forces of attraction between the rotor, shield and bearing may lead to excessive friction and thereby 
prevent micromotor operation. A possible model for this mechanism is described in [46]. In conjunction with the release process, micromotor operational characteristics may also be affected by the environment in which the micromotors operate $[37,39,47]$. For example, for micromotors with polysilicon/polysilicon mechanical contacts, operation in nitrogen rather than air enhances micromotor lifetime significantly $[38,39]$. However, for micromotors with at least one nitride/polysilicon mechanical contact $[13,35,36,45]$, lifetime is comparatively short and is not affected by operation in air or nitrogen. The effect of release and operational environment on micromotor performance requires further research to identify the competing mechanisms that affect friction, stiction and failure in these devices.

\subsection{Operational characteristics}

The overall operational characteristics of the side-drive micromotors reported to date are similar. However, the specific performance characteristics vary for the different micromotors. In general, the operational excitation voltages range from a few tens of volts for some micromotors [37-39] to near $100 \mathrm{~V}$ for others $[12-13,35,36$, $43,45]$. For the $1 \mu \mathrm{m}$ to $3 \mu \mathrm{m}$ air gaps used in the micromotors, the above excitation voltages correspond to electric field intensities above $10^{8} \mathrm{Vm}^{-1}$, which corroborates the initial estimates of breakdown in microscopic air gaps as discussed in section 3. The motive torque of the side-drive micromotors is typically of the order of $10 \mathrm{pN} \mathrm{m}$ (i.e. ten pico Newton meters).

Micromotor operation has only been studied under open-loop excitation. Under open-loop operation, a square-wave electric excitation is commutated on the stator; the stator phases are turned on one at a time. The excitation switching time is chosen to be larger than the settling time of the rotor transient. Therefore, micromotor operation has been of a stepping rather than continuous motion. For the different salient-pole micromotors reported to date, both underdamped [48-50] and overdamped [45] rotor transients have been observed. The rotor transient settling time has typically been under $250 \mu \mathrm{s}$ for these micromotors. For the salient-pole micromotors, open-loop stepping operational speeds of up to $15000 \mathrm{rpm}$ have been reported $[37,39]$. For the wobble micromotors, the rotor transient motion has not been measured; however, the open-loop stepping operational speeds have been as high as $700 \mathrm{rpm}[38,39]$. Typical wobble micromotor gear ratios have been in the range of 70 to 90 . In both cases, the maximum attainable speed has been limited by the power supply and not the micromotor.

For the micromotors reported to date, micromotor performance and life-time have varied significantly. For the first operational salient-pole micromotor [12], only a very slow stepping operation could be achieved at excitation voltages above $100 \mathrm{~V}$. This shortcoming was attributed to large electric forces attracting the rotor to the substrate. A conductive plate beneath the rotor and in electrical contact with it has been used in later designs to electrostatically shield the rotor from the substrate (as schematically demonstrated in figure 8). In these later micromotors, both performance and life-time have been improved significantly over time. Excitation voltages as low as $37 \mathrm{~V}$ and $26 \mathrm{~V}$ have been sufficient in operating some of the current salient-pole [37,39] and wobble $[38,39]$ micromotors, respectively. The open-loop operational speeds have been improved significantly as described above. Furthermore, micromotor life-time has been extended to many millions of cycles over a period of several days $[38,39]$; for these micromotors, a clear failure point has not yet been identified. Finally, repeatable micromotor operation has been achieved in the current micromotors [37-39]. However, both repeatability and reliability of micromotor operation are largely compromised by a lack of fundamental understanding of the mechanisms that affect micromotor operation.

\subsection{Friction}

Friction presents serious limitations to the performance and life-time of a micromotor; it presents a load to the micromotor which directly opposes useful work. During micromotor operation, the dynamic mechanical contacts at the bearing and the bushing (or the flange) result in friction. The bearing surfaces are subject to rolling contacts for the wobble micromotors and to sliding contacts for the salient-pole micromotors. For both micromotor types, the bushing surfaces are subject to sliding contacts. It is now well-documented that the bushing normal contact force is too large to be accounted for by the weight of the rotor alone $[33,36,37,39,44,45]$. This contact force is believed to be of electrostatic origin; an assertion which is corroborated by a comparison of results from frictional measurements in which no electric excitation is used [51]. In the latter case, rotor weight does account for the bushing normal contact force.

During wobble micromotor operation, the rotor is pulled against the bearing due to the normal electric force and rolls on the bearing. In this case, the resulting friction at the bearing contact is critical to the micromotor operation. However, for the salient-pole micromotors, bearing frictional forces are detrimental to micromotor performance. For the salient-pole micromotors, when the rotor is electrically grounded and is symmetrically centered in between a set of excited stator poles, the resultant of the radial forces on the rotor due to each excited stator pole is zero. However, when the rotor displaces radially, a net radial electric force (commonly referred to as a sidepull force $[45,48-50]$ ) develops which pushes the rotor into the bearing post. The side-pull force can result in significant friction in the bearing of salient-pole micromotors [45, 48-50].

Several studies have contributed to the understanding of friction in micromotors [31, 37-39, 45, 48-52]. These reported studies have so far focused almost exclusively on the friction of two polysilicon or polysilicon/silicon nitride surfaces sliding in contact with one another in an air or nitrogen environment. Differences between the frictional behavior of micro- 
motors with and without silicon nitride coatings in their bearings have been reported. For example, the operational characteristics of the micromotors described in [37-39], which have two contacting polysilicon surfaces, are significantly different for operation in air versus nitrogen. However, the micromotors described in $[12,13$, $35,36,43-45,48]$ that have at least one contacting silicon-nitride surface do not demonstrate a significant dependence on the operational environment. It is important to point out that micromotor failure is not necessarily due to wear but many times due to increased friction beyond the micromotor drive torque $[38,39,47]$.

The frictional characteristics of the micromotors are not only a function of materials but also a function of the micromotor release and operational environment $[37,39,47]$. With proper release, the frictional torques in the micromotors can be reduced to below that of the motive torque, yielding successful micromotor operation. Furthermore, the operational environment can affect friction in the micromotors significantly as described above. These results are in agreement with other frictional studies using macroscopic samples [53]. Surface microscopy techniques can be used in conjunction with experimental friction measurements to enhance our understanding of friction in micromotors. Surface studies can provide information on the chemical elements that may be present on the contact surfaces during micromotor operation. This approach has been effective in studying and enhancing the micromotor release process $[37,39]$.

Measurement of both dynamic and static friction is important in micromotors. In comparison to dynamic friction, static friction torques can be significantly larger in the micromotors $[37-39,45]$. For example, in one study, the resulting static friction torques resisting micromotor restart after $30 \mathrm{~s}$ at rest are found to be, on average, near $11 \mathrm{pN} \mathrm{m}$, which is a substantial fraction of the micromotor motive torque. These frictional forces were found to be independent of the bushing apparent area of contact in the experiments considered. Static friction torques exhibit a significant dependence on the duration of stationary contact. For micromotor restart after less than $1 \mathrm{~s}$ at rest, the frictional torques are a factor of two smaller than those for $30 \mathrm{~s}$ at rest [37-39].

To study static friction, studies have concentrated on estimating the motive torque necessary to initiate micro motor motion from rest $[36-39,48]$. This is accomplished by measuring the rotor position and the excitation voltage necessary to initiate motion from rest. This data is then used in conjunction with torqueposition curves for the micromotor which are calculated from electric field modeling in the micromotor. Note that accurate estimates of the friction torque requires an accurate micromotor torque-position analysis. Lateral resonant devices have also been used for static friction measurements [52]; the results are in agreement with the micromotor measurements.

To study dynamic friction, different measurement approaches have been used. In one study [51], passive gears and turbines have been spun with an air jet which is then shut off abruptly to measure the slow-down characteristics of the rotor. A simple dynamic model that accounts for viscous drag and coulomb friction is then used to estimate the dynamic coefficient of friction of the sliding contact surfaces. Dynamic coefficients of friction of 0.25 to 0.35 have been estimated for polysiliconpolysilicon sliding surfaces. In another study [48], a step excitation is used for actuating micromotors identical to that in figure 5 and the starting as well as stopping positions of the rotor are measured. From many such measurements, the resulting data can be used in conjunction with a dynamic model to estimate the dynamic coefficient of the sliding contact surfaces. In that study, dynamic coefficients of friction of 0.21 to 0.38 have been estimated for polysilicon--silicon nitride sliding surfaces.

Finally, a stroboscopic dynamometry technique has been used to measure the details of motion in salient-pole micromotors $[39,49,50]$. Again, the resulting data have been used in conjunction with a dynamic model to extract the frictional torque values. Dynamic frictional torques measured on micromotors similar to those for which the static friction torque values were given above, indicate values smaller than the static friction torques by at least one order of magnitude $[39,49,50]$. Dynamic friction effects were also found to be independent of the bushing apparent area of contact $[39,49,50]$. The stroboscopic dynamometry technique has been used in another study [45] to estimate the dynamic coefficient of sliding friction for polysilicon on silicon-nitride surfaces. The values.reported are in agreement with those above, from previous work [48].

\subsection{Wear}

Wear limits the lifetime of a micromotor in at least two ways. First, the bearing and bushings can wear down to the point where the micromotor ceases to function properly. This is particularly true of the bearing; its wear leads to greater rotor side pull and hence greater friction and wear. Second, as wear particles are generated, they can accumulate in the bearing where they cause it to bind, or they can be drawn electrostatically into the air gap where they accumulate and cause electric breakdown.

Wear in passive polysilicon microfabricated gears and turbines using an air-jet drive has been observed [51]. In that study, components were typically spun at $300.000 \mathrm{rpm}$ for an average of $3 \mathrm{~min}$ before significant wear at the bearing and the bushing was observed in SEM inspections. However, quantitative wear measurements were not possible and the drive forces were not known. The first in situ quantitative measurements of wear in micromotors were reported by studying changes in the gear ratio of wobble micromotors under extended operation [38].

For the wobble micromotors, the effect of wear on micromotor operation can be easily measured in situ, providing a convenient means for studying wear under electric excitation. Mechanical wear in the bearing of the wobble micromotor results in a corresponding increase 


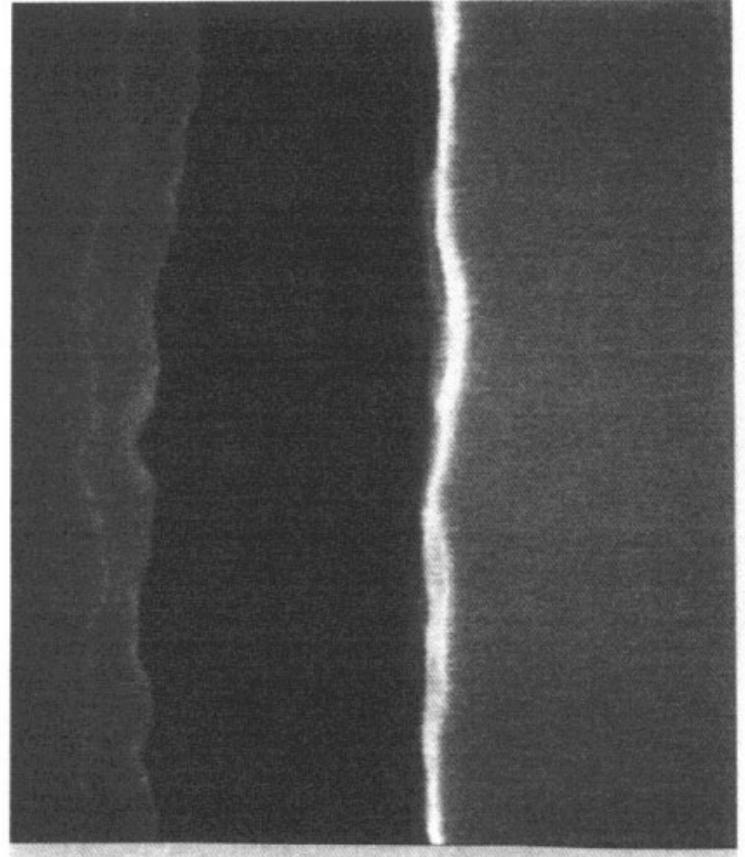

(a)

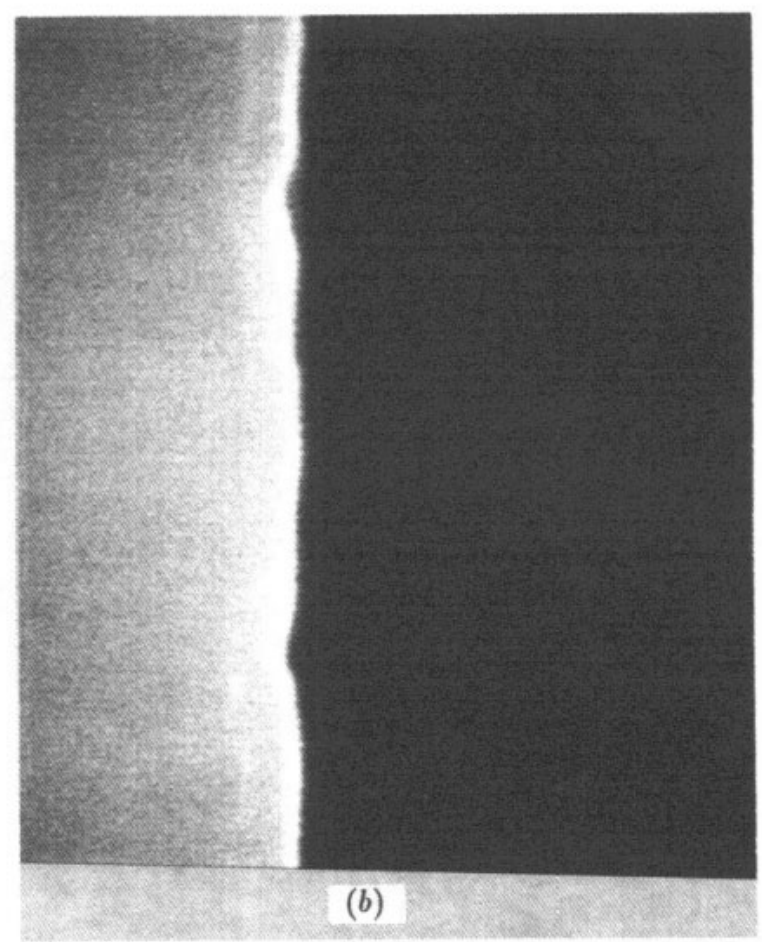

Figure 11. Magnified plan views from the rotor back side showing the rotor inner radius edges for two wobble micromotors [39]: (a) an unoperated wobble micromotor where the rotor inner radius edge is on the left, the bearing edge is on the right and the bearing clearance is the dark center area; (b) a wobble micromotor after near 30 million wobble cycles where the rotor back surface is on the left.

in the bearing clearance. Because the gear ratio of the wobble micromotor is equal to the bearing radius divided by the bearing clearance [38], under extended operation, changes in the gear ratio (reflected in changes in micromotor speed) can be a direct measure of wear in the bearing. This concept has been used to measure wear in the bearing of the wobble micromotors.

Extended operation of the whole micromotors to near 100 million wobble cycles were studied $[38,39]$ at excitation frequencies of $10.000 \mathrm{rpm}$ and $25.000 \mathrm{rpm}$, for operational durations of $150 \mathrm{~h}$ and $71 \mathrm{~h}$, respectively. The results indicate that bearing wear is significant and results in changes in the gear ratio of the wobble micromotors by as much as $20 \%$. Typical gear ratios are near 90 at the start of wobble micromotor operation and decrease to near 70 as the bearings wear out. The bearing wear is reflected in an increase of near $600 \AA$ in the bearing clearance. The associated bearing contact forces in these experiments have been near $1 \mu \mathrm{N}$. The bearing wear is attributed to the wearing out of the asperities of the rotor inner radius. These asperities result from the roughness of the RIE etched rotor inside radius sidewalls. Figure 11 shows the rotor inner radius from the back side for an unoperated wobble mictomotor and one that has been operated for 30 million wobble cycles.

For the salient-pole micromotors, wear particles are observed to form in the bearing and at the bushings with micromotor operation. In one study $[38,39]$, where the salient-pole micromotors have been operated at two rotor speeds of 160 and $2500 \mathrm{rpm}$ for a total of 40.000 rotor revolutions, SEM inspections of the bushing surfaces and the rotor inner radius revealed scattered wear particles several hundred angstroms in size. Figure 12 is a

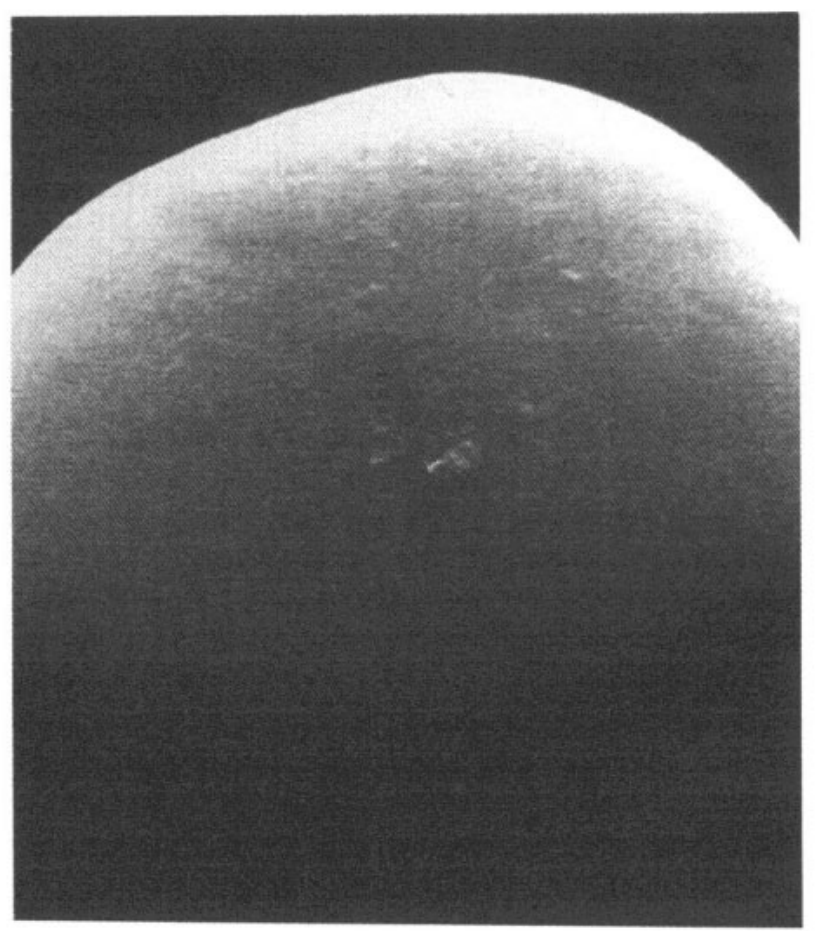

Figure 12. A magnified view of the bushing surface for a salient-pole side-drive micromotor after 40000 cycles of operation at $2500 \mathrm{rpm}$ [39]. 


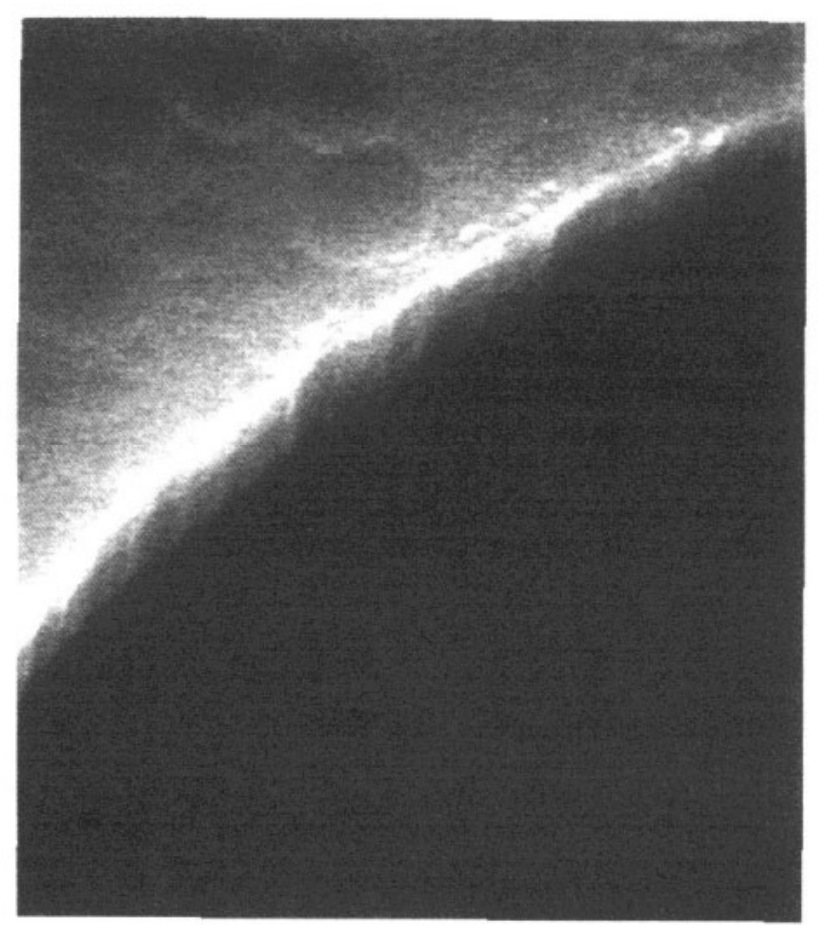

Figure 13. A portion of the rotor inner radius from the back side for the same micromotor as in figure 12 [39].

SEM photo showing a magnified view of the bushing surface which slides on the shield. Note the wear particles which adhere to the bushing surface. Wear on the polysilicon shield, which the bushings slide over during micromotor operation, could not be detected. Figure 13 is a SEM photo showing a portion of the rotor inner radius from the back side. Again, wear particles are observed adhering to the area adjacent to the bearing surface. Micromotors which have not yet been operated, when dismantled for SEM inspection, exhibit clean surfaces with no particles.

\section{Discussion}

Even though the electric micromotor technology has progressed rapidly, a number of micromotor performance limitations still exist. The most important of these performance limitations are small motive torque, friction problems and mechanical coupling. The micromotor technology is expected to expand in several directions including new designs, materials, fabrication techniques and applications. With the progress of the surface micromachining technology, it will become possible to implement new micromotor designs which imptove motive torque and reduce the frictional torques. At the same time, the surface micromachining techniques being developed for the micromotor technology are being applied to the development of alternative electric microactuators. One class of such microactuators has been the lateral resonant (or suspended) structures $[22,54,55]$; other microactuator designs are emerging $[56,57]$.
Both new materials and fabrication techniques can play a key role in the future progress of micromotor technology. New materials may be used to improve the friction and wear characteristics of the micromotors. At the same time, new fabrication techniques can provide a potential for new micromotor designs as well as improvements of the current designs. In one study, singlecrystalline silicon is being used in conjunction with silicon-glass bonding to fabrica te micromotors [32]. The use of tungsten as a structural material in surface micromachining processes similar to those above is also emerging [58]. Alternatively, the LIGA [59] and LIGA-type [60] processes provide a potential for fabrication of metallic micromotors that are significantly larger in the axial dimension than the surface micromachined micromotors but maintain comparable air-gap sizes. Therefore, micromotors with significantly larger motive torques can be produced.

Clearly, a necessary requirement for the future advance of the electric micromotor technology is a fundamental understanding of the operational, friction and wear characteristics. Without it, clear definition of future research and development is not possible. Toward the goal of establishing the fundamental knowledge necessary, theoretical modeling and experimental measurement techniques are required. Accurate modeling of the electric fields in the micromotor is needed for estimating the torque-position characteristics as well as the contact forces of electrostatic origin. The contact friction models must be refined to accurately predict the frictional behavior of the micromotors. Additional experimental measurement techniques as well as improvements of the currently documented experiments are necessary for further understanding of friction and wear in micromotors.

Finally, the electric micromotor technology can be applied to the fabrication of monolithic microrobotic systems. The micromotors can be incorporated in already existing micromechanisms to investigate the implementation of microrobotic systems [27]. A critical issue to be addressed in this area would be the mechanical coupling of the micromotors to other mechanisms on or off of the silicon wafer.

\section{Conclusion}

The brief review of the electric micromotor technology provided above indicates the rapid pace at which this technology is developing. In the past three to four years, the basic fabrication techniques, preliminary designs, operational characteristics, frictional behavior and wear properties have been studied. Reported operational micromotors have been of the rotary variablecapacitance salient-pole and harmonic (or wobble) sidedrive designs. These micromotors are capable of motive torques in the $10 \mathrm{pN}$ m order-of-magnitude range. Preliminary progress has been made in studying the operational, friction and wear characteristics of these devices. Typical operational voltages have been as low as $37 \mathrm{~V}$ 
and $26 \mathrm{~V}$ across $1.5 \mu \mathrm{m}$ air-gap salient-pole and harmonic micromotors. These excitations correspond to electric field intensities above $10^{8} \mathrm{~V} \mathrm{~m}^{-1}$ in the air gaps. Salient-pole and wobble micromotors have been reported to operate at speeds as high as $15.000 \mathrm{rpm}$ and $700 \mathrm{rpm}$, respectively. As described in section 3.6, micromotor life-times of at least many millions of cycles over a period of several days have been achieved. Friction and wear are important in micromotors and several techniques have been developed to experimentally measure them.

The progress made in the last three to four years has provided the foundation for future work toward improving and expanding the current micromotor technology. The micromotor technology is expected to expand in several directions including new designs, materials, fabrication techniques and applications. Progress in these areas is expected to improve the micromotor motive torque, friction and wear characteristics and mechanical coupling capabilities.

\section{References}

[1] Feury A M, Poteat T L and Trimmer W S 1986 A micromachined manipulator for submicron positioning of optical fibers IEEE Solid-State Sensor Workshop (Hilton Head, SC, 1986) Technical digest

[2] Mehregany M, Gabriel K J and Trimmer W S N 1987 Micro gears and turbines etched from silicon Sensors and Actuators 12 341-8

[3] Trimmer W S N and Gabriel K J 1987 Design considerations for a practical electrostatic micro motor Sensors and Actuators 11 189-206

[4] Fujita $\mathbf{H}$ and Omodaka A 1988 The fabrication of an electrostatic linear actuator by silicon micromachining IEEE Trans. Electron Devices ED$35731-4$

[5] Gabriel K J, Trimmer W S and Mehregany M Micro gears and turbines etched from silicon $4 \mathrm{th}$ Int. Conf. on Solid-State Sensors and Actuators (Tokyo, Japan, 1987) Technical digest pp 853-6

[6] Mehregany M, Gabriel K J and Trimmer W S N 1988 Integrated fabrication of polysilicon mechanisms IEEE Trans. Electron Devices ED-35 719-23

[7] Fan, L S, Tai Y C and Muller R S Pin joints, gears, springs, cranks, and other novel micromechanical structures 4th Int. Conf. on Solid-State Sensors and Actuators (Tokyo, Japan, 1987) Technical digest pp 849-52

[8] Fan L S, Tai Y C and Muller R S 1988 Integrated movable micromechanical structures for sensors and actuators IEEE Tran. Electron Devices ED-35 72430

[9] Bart S F, Lober T A, Howe R T, Lang J H and Schlecht M F 1988 Design considerations for micromachined electric actuators Sensors and Actuators 14 269-92

[10] Lober T A and Howe R T Surface-micromachining process for electrostatic microactuator fabrication IEEE Solid-State Sensor and Actuator Workshop (Hilton Head, SC, 1988) Technical digest pp 59-62

[11] Lober T A 1988 A microfabricated electrostatic motor design and process $M S c$ Thesis Massachusetts Institute of Technology, Cambridge, MA
[12] Fan L S, Tai Y C and Muller R S IC-processed electrostatic micro-motors IEEE Int. Electron Devices Meeting (San Francisco, CA, 1988) Technical digest pp 666-9

[13] Tai Y C, Fan L S and Muller R S IC-processed micromotors: design, technology, and testing Proc. IEEE Micro Electro Mechanical Systems Workshop (Salt Lake City, UT, 1989) pp 1-6

[14] Guckel H, Larsen S, Lagally M G and Wiley J D 1977 Electromechanical devices utilizing thin silicon diaphragms Appl. Phys. Lett. 31 618-9

[15] Lee Y S and Wise K D 1982 A batch-fabricated silicon capacitive pressure transducer with low temperature sensitivity IEEE Trans. Electron Devices ED-29 42-8

[16] Howe R T and Muller R S 1983 Polycrystalline silicon micromechanical beams J. Electrochem. Soc. 130 $1420-3$

[17] Howe R T 1984 Integrated Silicon Micromechanical Vapor Sensor PhD Thesis University of California, Berkeley

[18] Guckel H, Randazzo T and Burns D W 1985 A simple technique for the determination of mechanical strain in thin films with applications to polysilicon $J . A p p l$ Phys. 571671

[19] Greenwood J C 1984 Etched silicon vibrating sensor $J$. Phys. E: Sci. Instrum 17 650-2

[20] Mehregany M, Howe R T and Senturia S D 1987 Novel microstructures for the in-situ measurement of mechanical properties of thin films J. Appl. Phys. 62 3579-84

[21] Schmidt M A, Howe R T, Senturia S D and Haritonidis J H 1988 Design and calibration of a microfabricated floating-element shear-stress sensor IEEE Trans. Electron Devices ED-35 750-7

[22] Tang W C, Nguyen T H and Howe R T 1989 Laterally driven polysilicon resonant microstructures Sensors and Actuators 20 25-32

[23] Nathanson H C, Newell W E, Wickstrom R A and Davis J R J 1967 The resonant gate transistor IEEE Trans. on Electron Devices ED-14 117-33

[24] Muller R S 1987 From ICs to microstructures: materials and technologies Proc. IEEE Micro Robots and Teleoperators Workshop (Hyannis, MA, 1987)

[25] Schmidt M A 1988 Microsensors for the Measurement of Shear Forces in Turbulent Boundary Layers $P h D$ Thesis Massachusetts Institute of Technology, Cambridge, MA

[26] Guckel H, Burns D W and Rutigliano C R 1986 Design and construction techniques for planar polysilicon pressure transducers with piezoresistive read-out IEEE Solid-State Sensor Workshop (Hilton Head, SC, 1986) Technical digest

[27] Behi F, Mehregany M and Gabriel K J A microfabricated three-degree-of-freedom parallel mechanism Proc. IEEE Micro Electro Mechanical Systems Workshop (Napa Valley, CA, 1990) pp 159-65

[28] Lang J H, Schlecht M F and Howe R T Electric micromotors: electromechanical characteristics Proc. IEEE Micro Robots and Teleoperators Workshop (Hyannis, MA, 1987) (reprinted in Proc. ASME Annual Winter Meeting (Boston, MA, 1987) DSC-6 pp 403-10)

[29] Paschen F 1889 Ueber die zum Funkenübergang in Luft, Wasserstoff und Kohlensäure bei verschiedenen Drucken erforderliche Potentialdifferenz Ann. Phys. Lpz 37 69-96

[30] Bart S F and Lang J H 1989 An analysis of electroquasistatic induction micromotors Sensors and Actuators 20 97-106

[31] Bart S F 1990 Modeling and design of electroquasistatic microactuators $P h D$ Thesis 
Massachusetts Institute of Technology, Cambridge, MA

[32] Suzuki K and Tanigawa H 1991 Single crystal silicon rotational micromotors Proc. IEEE Micro Electro Mechanical Systems Workshop (Nara, Japan, 1991) pp $15-20$

[33] Mehregany M, Bart S F, Tavrow L S, Lang J H, Senturia S D and Schlecht M F 1990 A study of three microfabricated variable-capacitance motors Sensors and Actuators A21-23 173-9

[34] Tavrow L S, Bart S F, Lang J H and Schlecht M F 1990 A Locos process for an electrostatic microfabricated motor Sensors and Actuators A2123 893-8

[35] Tai Y C and Muller R S 1989 IC-processed electrostatic synchronous micromotors Sensors and Actuators 20 $49-55$

[36] Tai Y C 1989 IC-processed polysilicon micromechanics: technology, material, and devices $P h D$ Thesis University of California, Berkeley, CA

[37] Mehregany M, Nagarkar P, Senturia S D and Lang J $\mathrm{H}$ Operation of microfabricated harmonic and ordinary side-drive motors Proc. IEEE Micro Electro Mechanical Systems Workshop (Napa Valley, CA 1990) pp 1-8

[38] Mehregany M, Senturia S D and Lang J H Friction and wear in microfabricated harmonic side-drive motors IEEE Solid-State Sensor and Actuator Workshop (Hilton Head, SC, 1990) Technical digest pp 17-22

[39] Mehregany M 1990 Microfabricated Silicon Electric Mechanisms PhD Thesis Massachusetts Institute of Technology, Cambridge, MA

[40] Mehregany M, Bart S F, Tavrow L S, Lang J H and Senturia S D 1990 Principles of design and fabrication of variable-capacitance side-drive micromotors J. Vac. Sci. Technol. A8 3614-24

[41] Trimmer W and Jebens R 1989 Harmonic electrostatic motors Sensors and Actuators 20 17-24

[42] Jacobsen S C, Price R H, Wood J E, Rytting T H and Rafaelof M 1989 A design overview of an eccentricmotion electrostatic microactuator Sensors and Actuators 20 1-16

[43] Fan L S, Tai Y C and Muller R S 1989 1C-processed electrostatic micromotors Sensors and Actuators 20 41-7

[44] Fan L S 1989 Integrated micromachinary: moving structures on silicon chips $P h D$ Thesis University of California, Berkeley, CA

[45] Tavrow L S 1991 A Locos-Based Microfabricated Radial-Gap Electric Motor PhD Thesis Massachusetts Institute of Technology, Cambridge, MA

[46] Lang J H 1989 Initial thoughts on the dynamics and control of electric micromotors Proc. 3rd Toyota Conference on Integrated Micro Motion Systems (Nissin, Aichi, Japan, 1989) pp 9/1-9/14

[47] Mehregany M, Phillips S M, Hsu E T and Lang $J$ H 1991 Operation of harmonic side-drive micromotors studied through gear ratio measurements 6 th Int. Conf. Solid-State Sensors and Actuators (San Francisco, CA, 1991) Technical digest

[48] Tai Y C and Muller R S 1990 Frictional study of ICprocessed micromotors Sensors and Actuators A2123 180-3

[49] Bart S F, Mehregany M, Tavrow L S, Lang J H and Senturia S D 1990 Measurements of electric micromotor dynamics Proc. ASME Winter Annual Meeting (Dallas, TX, 1990) DSC 19 19-29

[50] Bart S F, Mehregany M, Tavrow L S, Lang J H and Senturia S D 1991 Electric micromotor dynamics Trans. Electron Devices at press

[51] Gabriel K J, Behi F, Mahadevan R and Mehregany $\mathbf{M}$ 1990 In situ measurements of friction and wear in integrated polysilicon microstructures Sensors and Actuators A21-23 184-8

[52] Lim M G, Chang J C, Schultz D P, Howe R T and White R M 1990 Polysilicon microstructures to characterize static friction Proc. IEEE Micro Electro Mechanical Systems Workshop (Napa Valley, CA 1990) pp 82-8

[53] Deng K, Ko W H and Michal G A preliminary study on friction measurements in MEMS 6th Int. Conf. Solid-State Sensors and Actuators (San Francisco, $C A, 1991)$ Technical digest

[54] Tang W C, Nguyen T H, Judy M W and Howe R T 1990 Electrostatic-comb drive of lateral polysilicon resonators Sensors and Actuators A21-23 328-31

[55] Brennan R A, Pisano A P and Tang W C 1990 Multiple mode micromechanical resonators Proc. JEEE Micro Electro Mechanical Systems Workshop (Napa Valley, CA, 1990) pp 9-14

[56] Kim C J, Pisano A P, Muller R S and Lim M G Polysilicon microgripper IEEE Solid-State Sensor and Actuator Workshop (Hilton Head, SC, 1990) Technical digest pp 48-51

[57] Moroney R M, White R M and Howe R T 1990 Ultrasonic micromotors: physics and applications Proc. IEEE Micro Electro Mechanical Systems Workshop (Napa Valley, CA 1990) pp 182-7

[58] Chen L Y, Zhang Z L, Yao J J, Thomas D C and MacDonald N C Selective chemical vapor deposition of tungsten for microdynamic structures Proc. IEEE Micro Electro Mechanical Systems Workshop (Salt Lake City, UT, 1989) pp 82-7

[59] Ehrfeld W, Bley P, Gotz F, Hagmann P, Maner A, Mohr J, Moser H O, Munchmeyer D, Schelb W, Schmidt D and Becker E B 1987 Fabrication of microstructures using the LIGA process Proc. IEEE Microrobots and Teleoperators Workshop (Hyannia, $M A, 1987$ )

[60] Guckel H, Christenson T R, Skrobis K J, Denton D D, Choi B, Lovell E G, Lee J W, Bajikar S S and Chapman T W Deep X-ray and UV lithographies for micromechanics IEEE Solid-State Sensor and Actuator Workshop (Hilton Head, SC, 1990) Technical digest pp 118-22 